\title{
Sub-THz Photonic-Transmitters Based on Separated-Transport-Recombination Photodiodes and a Micromachined Slot Antenna
}

\author{
Yu-Tai Li, J.-W. Shi, Ci-Ling Pan, C.-H. Chiu, W.-S. Liu, Nan-Wei Chen, C.-K. Sun, and J.-I. Chyi
}

\begin{abstract}
We demonstrate a novel photonic transmitter, which is composed of a low-temperature-grown GaAs (LTG-GaAs)-based separated-transport-recombination photodiode and a micromachined slot antenna. Under femtosecond optical pulse illumination, this device radiates strong electrical pulses $(4.5-\mathrm{mW}$ peak power) without the use of a Si-lens. It can be observed in the Fourier transform infrared spectrometer spectrum of radiated pulses that a significant resonance, with a peak power of approximately $300 \mu \mathrm{W}$, occurs at $500 \mathrm{GHz}$, which corresponds to the designed resonant frequency of the slot antenna. The saturation problem related to the output terahertz power that occurs with the traditional LTG-GaAs-based photonic-transmitters when operated under high external applied electrical fields $(>50 \mathrm{kV} / \mathrm{cm})$ has been eliminated by the use of our device.
\end{abstract}

Index Terms-Low-temperature-grown GaAs (LTG-GaAs), photonic transmitter, terahertz ( $\mathrm{THz})$.

\section{INTRODUCTION}

$\mathbf{R}$ ECENTLY, terahertz (THz) technology has gained considerable attention, but a compact $\mathrm{THz}$ emitter is critical for it to be applied. There are several millimeter- or submillimeter-wave emission techniques that have already been introduced, such as Gunn diodes or resonant tunneling diodes [1], p-type Ge-based or quantum cascade $\mathrm{THz}$ lasers [2], [3], and photonic transmitters [4]-[8], which are composed of ultrahigh-speed photodetectors and printed-circuit antennas. Among these proposed techniques, photonic transmitters have the advantages of simplicity, room-temperature operation, tunable $\mathrm{THz}$ wavelength, and integrability with other semiconductor devices, resulting in compact $\mathrm{THz}$ sources. Both low-temperature-grown GaAs (LTG-GaAs) [7], [8] and Uni-traevling-carrier photodiode (UTC-PD) [5], [6] based photonic-transmitters have captured attention due to their excellent high-speed and high-power performance. However, under high reverse bias voltages, both types of devices usually suffer from saturation of the output $\mathrm{THz}$ power. Such a phenomenon can be attributed to the intervalley scattering

Manuscript received October 4, 2006; revised February 27, 2007

Y.-T. Li and C.-L. Pan are with the Department of Photonics and Institute of Electro-Optical Engineering, National Chiao Tung University, Hsinchu, 300, Taiwan, R.O.C.

J.-W. Shi, C.-H. Chiu, W.-S. Liu, N.-W. Chen, and J.-I. Chyi are with the Department of Electrical Engineering, National Central University, Taoyuan, 320 Taiwan, R.O.C. (e-mail: jwshi@ee.ncu.edu.tw).

C.-K. Sun is with the Graduate Institute of Electro-Optical Engineering, National Taiwan University, Taipei 106, Taiwan, R.O.C.

Digital Object Identifier 10.1109/LPT.2007.897439 of photogenerated electrons [6] and the life-time increasing effect [7], [9], for the cases of UTC-PDs and LTG-GaAs based photodetectors, respectively. On the other hand, for most reported photonic-transmitters operating in the millimeter and submillimeter wavelength regimes, Si lenses are usually required to increase the antenna radiation efficiency [4]-[6], [8]. However, this integration will not only increase the cost of packaging but decrease some of the $\mathrm{THz}$ radiation due to coupling loss in the dielectric material. In addition, the distance from the center to the edge of the Si-lens also creates an obstacle to the optical beam when coupled into side-illumination photodetectors. In this letter, we demonstrate a novel photonic transmitter, which is composed of an LTG-GaAs-based edge-coupled separated-transport-recombination PD (STR-PD) [10] and a micromachined coplanar-waveguide (CPW) fed slot antenna. Under femtosecond optical pulse illumination, this device radiates strong electrical pulses (4.5-mW peak power) without using a Si-lens. A significant resonance (with peak power of around $300 \mu \mathrm{W}$ ) which occurs at $500 \mathrm{GHz}$, can be observed in the Fourier transform infrared spectrometer (FTIR) spectrum of radiated electrical pulses. It corresponds to the designed resonant frequency of the slot antenna. By adopting the LTG-GaAs-based STR-PD as the active part of our transmitter, the reported saturation phenomenon of traditional LTG-GaAs-based photonic-transmitter, under high applied external electrical fields, [7]-[9] has also been eliminated.

\section{Device Structure}

The top view of the demonstrated $\mathrm{THz}$ photonic-transmitter is shown in Fig. 1. It is composed of a high-speed edge-coupled STR-PD [10], which has CPW-type traveling-wave electrodes [11], a radio-frequency (RF) isolation bias tee, and a CPW fed slot antenna [7], [12]. We adopt this kind of antenna as our radiator because the active STR-PD is easy to integrate and has a higher radiation power at the designed resonant frequency than do the spiral antennas. The RF isolation bias tee, which operates as an inductance, makes it possible to avoid high-frequency ac current leakage into the dc probe pad and decreases the radiation efficiency. By using the techniques of selective wet chemical etching and back-side lithography, a fraction of the GaAs substrate below the slot antenna can be removed. The fabricated slot antenna will stand on the AlGaAs membrane with a 5- $\mu$ m thickness. Except for the membrane slot antenna, the remaining area of the photonic-transmitter chip is still above the robust GaAs substrate which is capable of enduring the wire-bonding process 


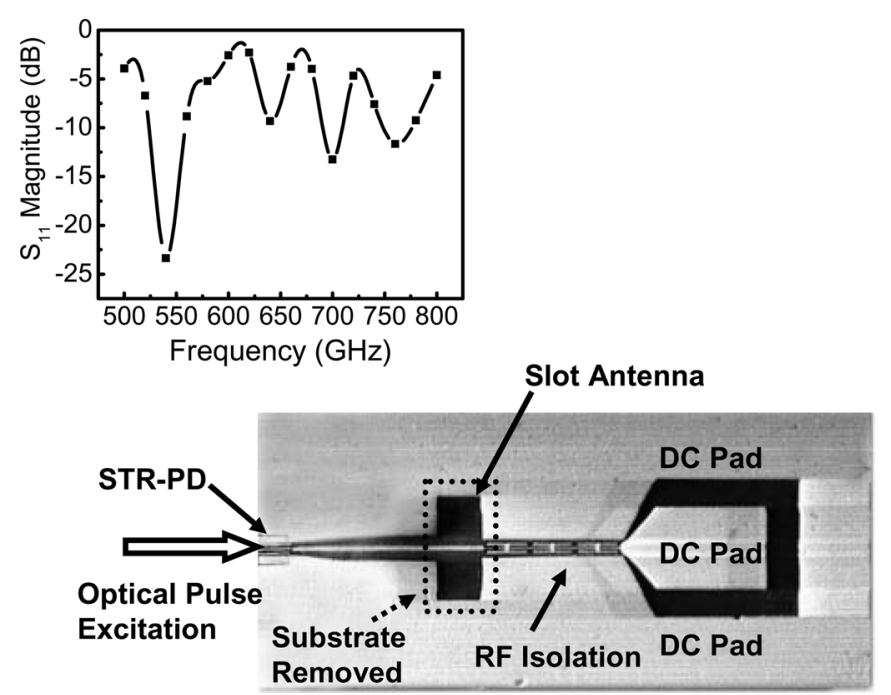

Fig. 1. Top-view of the fabricated photonic-transmitter. The left-hand arrow indicates the direction of the input optical pulse. The inset shows the simulated frequency response of the slot antenna. A significant resonance can be observed around the 500-GHz operating frequency.

necessary for device packaging. With a relatively thin membrane $(\sim 5 \mu \mathrm{m})$, compared with the operating wavelength (hundreds of micrometers) of the THz signal, the problem of the substrate mode can be eliminated [12]. A higher radiation efficiency of our membrane antenna (without requiring integration with a Si-lens), as compared to other $\mathrm{THz}$ printed-circuit antennas fabricated without thinning the substrate and integrating additional optic elements, can be expected. The inset to Fig. 1 shows the simulated frequency response of our transmitter without considering the influence of active PDs. We can clearly see that the designed resonant frequency of the slot antenna is around $500 \mathrm{GHz}$. The active part of our demonstrated transmitter is an STR-PD, and has been demonstrated in our previous work [10]. An LTG-GaAs layer with an extremely short carrier lifetime is inserted at the center of its active region to serve as a recombination center and the two surrounding high-quality GaAs-based depletion layers serve as depleted transport layers [10]. Compared with the traditional p-i-n PD, the STR-PD minimizes the space-charge-screening effect under high-power operation and exhibits much better speed and output power performance during both pulse-mode (PM) and continuous-wave operations [10], [13].

\section{EXPERIMENTAL METHODS AND RESULTS}

We directly excited our device using a femtosecond Ti : sapphire $(\lambda=800 \mathrm{~nm})$ mode-locked laser, then measured the radiated impulse responses of the transmitter. The excitation optical plus, which has an $82-\mathrm{MHz}$ repetition rate and a 100 -fs pulsewidth, was focused by an objective lens on the input facets of the device. We used two parabolic mirrors to collimate and focus the radiated $\mathrm{THz}$ pulse onto a liquid-helium-cooled $\mathrm{Si}$ bolometer for power measurement. The responsivity of the $\mathrm{Si}$ bolometer was calibrated with a blackbody radiation source [7]. We also measured and calibrated the radiated $\mathrm{THz}$ beam propagation loss in air by measuring the $\mathrm{THz}$ intensity as a function of the propagation distance. The propagation loss was about (a)

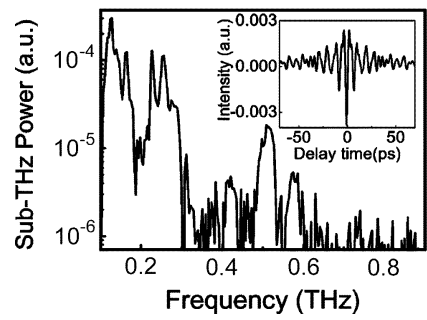

(b)

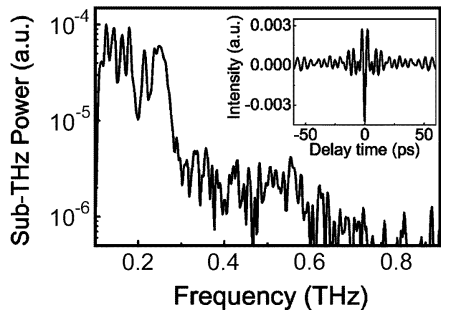

Fig. 2. Fourier-transformed spectra of our photonic-transmitters with different active lengths: (a) 23 and (b) $60 \mu \mathrm{m}$. The insets show the original interference spectrum in the time domain measured using the FTIR system. (a)

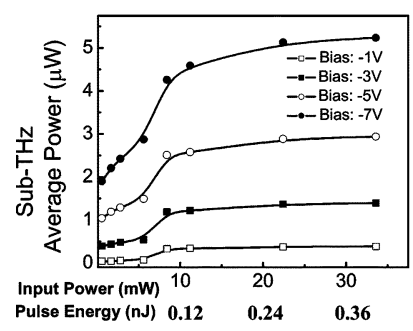

(b)

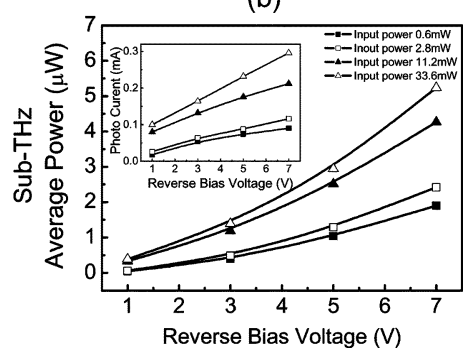

Fig. 3. Measured sub-THz intensity versus (a) optical pumping power (pulse energy) and (b) reverse bias voltages for our photonic-transmitters with a 23- $\mu \mathrm{m}$ active length. The inset to (b) shows the measured output photocurrent versus reverse bias voltages under different optical pumping powers.

$0.082 \mathrm{~cm}^{-1}$. The RF spectra and waveforms of radiated signal were measured by a Martin-Puplett-type FTIR [14] with the same Bolometer as in the power measurement The FTIR system consisted of two wire grid polarizers, the first one used as a reflector at the input port of system and the second one acting as a beam splitter. By scanning the movable retro-reflector, the interference spectrum (waveform) of the input sub-THz wave in the time domain could be obtained. The frequency responses of the radiated signal could thus be obtained through the use of Fourier-transform techniques. The measured interference patterns and corresponding transformed spectra of devices with a $23-\mu \mathrm{m}$ and a $60-\mu \mathrm{m}$ active lengths and the same $2-\mu \mathrm{m}$ waveguide width are shown in Fig. 2(a) and (b), respectively. In the transformed spectra, the frequency components below $100 \mathrm{GHz}$ have been truncated due to the cutoff frequency of our bolometer $(<100 \mathrm{GHz})$. Both devices exhibited significant resonance at around $150-$ and $250-\mathrm{GHz}$ frequency. A significant resonance at the designed resonant frequency $(\sim 500 \mathrm{GHz})$ of the slot antenna was only observed for a device with a short active length, as shown in Fig. 2(a). The device with a long active length should have a poorer speed performance and lower output power in the high frequency regime than a short device. A much less apparent resonance at the designed frequency $(\sim 500 \mathrm{GHz})$ of the long device was indeed observed. The parasitic oscillations in the $0.15-$ and $0.25-\mathrm{THz}$ frequency regime may be attributed to the influence of the integrated PD, which was not included in our antenna design. Fig. 3(a) shows the measured sub-THz power versus injected optical power of devices with a $23-\mu \mathrm{m}$ active length under different reverse bias voltages. When the average injected optical power was $\sim 30 \mathrm{~mW}$, as much as $5 \mu \mathrm{W}$ of total average sub-THz power 
was measured, which could be calibrated as around $4.3-\mathrm{dB}$ propagation loss after 12-cm propagation length. Based on the maximum radiated average power, the FTIR spectrum, and the full-width at half-maximum (FWHM) of the radiated electrical pulse envelope $(\sim 15 \mathrm{ps})$ with a near Gaussian distribution, as shown in Fig. 2(a) and its inset, we can roughly calculate the radiated total peak power and peak power at $500-\mathrm{GHz}$ resonant frequency, which is around $4.5 \mathrm{~mW}$ and $300 \mu \mathrm{W}$, respectively. In addition, the output sub-THz power shows a near parabolic relation between the injected average optical powers when its value is below $10 \mathrm{~mW}(-7 \mathrm{~V}$ bias), which corresponds to a 122-pJ injected optical pulse energy. On the other hand, when the injected pulse energy is over this value, saturation phenomenon of the sub-THz power can be clearly observed. The achieved saturation injected optical pulse energy $(122 \mathrm{pJ} / \mathrm{pulse})$ and corresponding peak-output-photocurrent (184 mA), roughly estimated from the average photocurrent and the FWHM of radiated electrical pulsewidth $(\sim 15 \mathrm{ps})$ with a near Gaussian distribution, is much higher than those of reported UTC-PD (1 pJ/pulse and $76.8 \mathrm{~mA})$ with a close device active area $\left(\sim 20 \mu \mathrm{m}^{2}\right)$ under PM operation [15].

The measured bias-dependent sub-THz power of the device with a $23-\mu \mathrm{m}$ active length, under different optical pumping powers, is shown in Fig. 3(b). The inset shows the measured photocurrent versus de reverse bias under different optical pumping powers. The maximum reverse bias voltage $(-7 \mathrm{~V})$ is limited by the device failure. The poor dc responsivity (0.12 A/W, 0.6-mW injected optical power and $-7 \mathrm{~V}$ bias) of our primary demonstrated device is limited by the poor coupling efficiency between a narrow waveguide with a $2-\mu \mathrm{m}$ width and the injected optical signal with around $\sim 5-\mu$ m beam radius. A higher responsivity performance can be expected by improving the optical waveguide design or using an objective with a higher numerical aperture to focus the injected light onto the input facet of the device. Regarding the reported LTG-GaAs-based photodetectors and photomixers [7]-[9], they usually suffer from serious bandwidth degradation and saturation of radiated $\mathrm{THz}$ power under high reverse bias voltage and high external applied electric field $(>50 \mathrm{kV} / \mathrm{cm})$ due to the lifetime increasing effect of LTG-GaAs [9]. However, for our devices even under the highest reverse bias $(-7 \mathrm{~V})$, corresponding to an external applied electric field of up to $200 \mathrm{kV} / \mathrm{cm}$ in the active region, no radiated power saturation phenomenon could be observed. Furthermore, the output sub-THz power has an ideally quadric relation with the reverse bias voltages, due to the fact that the output photocurrent is linearly proportional to the reverse bias voltage, as shown in the inset. The superior bias dependent performance of our transmitter to the traditional LTG-GaAs-based photomixer is made possible because, for our STR-PD, the external applied electric field is concentrated in the two high-quality GaAs transport layers instead of the LTG-GaAs-based recombination center [10]; the lifetime increasing effect of the LTG-GaAs layer under the high electric field $(>50 \mathrm{kV} / \mathrm{cm})$ can thus be neglected.

\section{CONCLUSION}

We have demonstrated a novel photonic-transmitter under PM operations, composed of an STR-PD and a micromachined slot antenna but without the integration with a Si-lens. A strong sub- $\mathrm{THz}$ radiation was measured at the designed resonant frequency $(\sim 500 \mathrm{GHz})$ by the FTIR system. Furthermore, the saturation problem of the radiated $\mathrm{THz}$ power, that occurs when a traditional LTG-GaAs-based photomixer is operated under a high reverse bias voltage, has been eliminated. These promising electrical-optical measurement results point out the advantages of our device for application as a compact $\mathrm{THz}$ source that can be integrated with semiconductor lasers.

\section{REFERENCES}

[1] H. Eisele, A. Rydberg, and G. I. Haddad, "Recent advances in the performance of InP Gunn devices and GaAs TUNNET diodes for the 100-300 GHz frequency range and above," IEEE Trans. Microw. Theory Tech., vol. 48, no. 4, pt. 2, pp. 626-631, Apr. 2000.

[2] Y. P. Gousev, I. V. Altukhov, K. A. Korolev, V. P. Sinis, M. S. Kagan, E. E. Haller, M. A. Odnoblyudov, I. N. Yassievich, and K.-A. Chao, "Widely tunable continuous-wave THz laser," Appl. Phys. Lett., vol. 75, pp. 757-759, Aug. 1999.

[3] S. Barbieri, J. Alton, S. S. Dhillon, H. E. Beere, M. Evans, E. H. Linfield, A. G. Davies, D. A. Ritchie, R. Kohler, A. Tredicucci, and F. Beltram, "Continuous-wave operation of terahertz quantum-cascade lasers," IEEE J. Quantum Electron., vol. 39, no. 4, pp. 586-591, Apr. 2003.

[4] A. Stohr, A. Malcoci, A. Sauerwald, I. C. Mayorga, R. Güsten, and D. S. Jäger, "Ultra-wide-band traveling-wave photodetectors for photonic local oscillators," J. Lightw. Technol., vol. 21, no. 12, pp. 3062-3070, Dec. 2003.

[5] H. Ito, F. Nakajima, T. Furuta, and T. Ishibashi, "Continuous THzwave generation using antenna-integrated uni-travelling-carrier photodiodes," Semicond. Sci. Technol., vol. 20, pp. S191-S1918, Jun. 2005.

[6] H. Ito, T. Furuta, F. Nakajima, K. Yoshino, and T. Ishibashi, "Photonic generation of continuous $\mathrm{THz}$ wave using uni-traveling-carrier photodiode," J. Lightw. Technol., vol. 23, no. 12, pp. 4016-4021, Dec. 2005.

[7] M.-C. Tien, H. H. Chang, J. Y. Lu, L. J. Chen, S. Y. Chen, R. B. Wu, W. S. Liu, J. I. Chyi, and C. K. Sun, "Device saturation behavior of submillimeter-wave membrane photonic transmitters," IEEE Photon. Technol. Lett., vol. 16, no. 3, pp. 873-875, Mar. 2004.

[8] S. M. Duffy, S. Verghese, K. A. McIntosh, A. Jackson, A. C. Gossard, and S. Matsuura, "Accurate modeling of dual dipole and slot elements used with photomixers for coherent terahertz output power," IEEE Trans. Microw. Theory Tech., vol. 49, no. 6, pt. 1, pp. 1032-1038, Jun. 2001.

[9] N. Zamdmer, Q. Hu, K. A. Mclntosh, and S. Verghese, "Increase in response time of low-temeprature-grown GaAs photoconductive switches at high voltage bias," Appl. Phys. Lett., vol. 75, pp. 2313-2315, Oct. 1999.

[10] J. W. Shi, H. C. Hsu, F.-H. Huang, W. S. Liu, J. I. Chyi, J.-Y. Lu, C.-K. Sun, and C.-L. Pan, "Separated-transport-recombination p-i-n photodiode for high-speed and high-power performance," IEEE Photon. Technol. Lett., vol. 17, no. 8, pp. 1722-1724, Aug. 2005.

[11] K. S. Giboney, M. J. W. Rodwell, and J. E. Bowers, "Traveling-wave photodetector theory," IEEE Trans. Microw. Theory Tech., vol. 45, no. 8, pt. 2, pp. 1310-1319, Aug. 1997.

[12] K. C. Gupta, R. Garg, I. Bahl, and P. Bhartia, Microstrip Lines and Slotlines. Boston, London: Artech House, 1996.

[13] J. W. Shi, Y.-T. Li, C.-L. Pan, M. L. Lin, Y. S. Wu, W. S. Liu, and J. I. Chyi, "Separated-transport-recombination p-i-n photodiode (STR-PD) with high-speed and high-power performance under continuous-wave (CW) operation," in Conf. Laser and Electro-Optics (CLEO/QELS 2006), 2006, OSA Tech. Dig., Paper CTuS6.

[14] D. H. Martin and E. Puplett, "Polarised interferometric spectrometry for the millimeter and submillimeter spectrum," Infared Phys., vol. 10, pp. 105-109, Jun. 1970.

[15] N. Shimizu, N. Watanabe, T. Furuta, and T. Ishibashi, "InP-InGaAs Uni-traveling-carrier photodiode with improved 3-dB bandwidth of over $150 \mathrm{GHz}$," IEEE Photon. Technol. Lett., vol. 10, no. 3, pp. 412-414, Mar. 1998. 